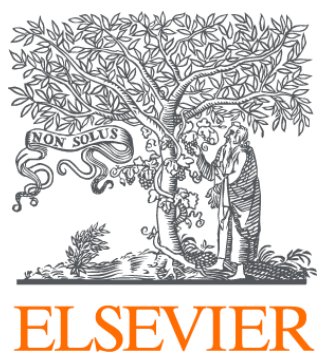

Since January 2020 Elsevier has created a COVID-19 resource centre with free information in English and Mandarin on the novel coronavirus COVID-

19. The COVID-19 resource centre is hosted on Elsevier Connect, the company's public news and information website.

Elsevier hereby grants permission to make all its COVID-19-related research that is available on the COVID-19 resource centre - including this research content - immediately available in PubMed Central and other publicly funded repositories, such as the WHO COVID database with rights for unrestricted research re-use and analyses in any form or by any means with acknowledgement of the original source. These permissions are granted for free by Elsevier for as long as the COVID-19 resource centre remains active. 


\title{
The drop in reported invasive pneumococcal disease among adults during the first COVID-19 wave in the Netherlands explained.
}

\author{
Kirsten K.T. Dirkx ${ }^{1,2}$, Bert Mulder ${ }^{1}$, Annelies S. Post ${ }^{1}$, Martijn H. Rutten ${ }^{3}$, \\ Caroline M.A. Swanink ${ }^{4}$, Heiman F.L. Wertheim ${ }^{2}$, Amelieke J.H. Cremers ${ }^{2}$ \\ ${ }^{1}$ Department of Clinical Microbiology and Infectious Diseases, Canisius-Wilhelmina Ziekenhuis, Nijmegen, the Netherlands \\ ${ }^{2}$ Department of Clinical Microbiology, Radboud Centre for Infectious Diseases, Radboudumc, Nijmegen, the Netherlands \\ ${ }^{3}$ Department of Primary and Community Care, Radboud University Medical Centre, Nijmegen, The Netherlands \\ ${ }^{4}$ Department of Clinical Microbiology and Immunology, Rijnstate, Arnhem, the Netherlands
}

\section{A R T I C L E I N F O}

\section{Article history:}

Received 1 August 2021

Revised 22 August 2021

Accepted 23 August 2021

\section{Keywords:}

S. pneumoniae

IPD

COVID-19

Surveillance

Pandemic

\begin{abstract}
A B S T R A C T
Objectives: Streptococcus pneumoniae is the leading bacterial pathogen causing respiratory infections. Since the COVID-19 pandemic emerged, less invasive pneumococcal disease (IPD) was identified by surveillance systems worldwide. Measures to prevent transmission of SARS-CoV-2 also reduce transmission of pneumococci, but this would gradually lead to lower disease rates. Design: Here, we explore additional factors contributing to the instant drop in pneumococcal disease cases captured in surveillance. Results: Our observations on referral practices and other impediments to diagnostic testing indicate that residual IPD has likely occurred but remained undetected by conventional hospital-based surveillance. Conclusions: Depending on the setting, we discuss alternative monitoring strategies that could improve understanding of pneumococcal disease dynamics.

(c) 2021 The Authors. Published by Elsevier Ltd on behalf of International Society for Infectious Diseases. This is an open access article under the CC BY license (http://creativecommons.org/licenses/by/4.0/)
\end{abstract}

\section{Background}

The bacterium Streptococcus pneumoniae (the pneumococcus) is the leading cause of community-acquired pneumonia and meningitis worldwide (G.B.D. Lower Respiratory Infections Collaborators, 2018; van de Beek et al., 2016). Children under five and adults above 65 are most at risk for pneumococcal infections (van der Poll and Opal, 2009). Mortality among hospitalized patients is $10 \%$ to $15 \%$. Lower respiratory tract infections are the primary cause of sepsis (Klein Klouwenberg et al., 2015, Leligdowicz et al., 2014, Vincent et al., 2006) and account for the largest proportion of hospitalizations due to infectious diseases (Zorginstituut Nederland en Zinnige, 2019), even before the COVID-19 pandemic. In less than $10 \%$ of all pneumococcal infections in the Netherlands, the pathogen is identified by culture of a normally sterile site like blood or cerebrospinal fluid, which is called invasive pneumococcal disease (IPD) (Gezondheidsraad, 2018). This percentage is likely an underestimation of the prevalence of bacteremia among adults with a pneumococcal infection. Collecting blood cultures is often limited to patients who present at the hospital with fever and severe respiratory disease. However, of captured pneumococcal bacteremias, $29 \%$ occurred in patients with normal body temperature, and $30 \%$ were classified as mild pneumonia (this study). Furthermore, pneumococci are fragile bacteria whose culture is eas- ily disturbed by prior antibiotics use (Endeman et al., 2008, RuizGonzalez et al., 1999).

Because of the introduction of pediatric pneumococcal vaccination programs, the incidence of IPD is under surveillance in many countries. During the first COVID-19 wave in the Netherlands, an $80 \%$ drop in IPD was reported by the Netherlands Reference Laboratory of Bacterial Meningitis (NRLBM) (RIVM, 2020a). In three hospitals in Eastern Netherlands, we recognized this trend and additionally noted a high mortality among adult IPD patients. To understand these observations, we compared the IPD cases during the first COVID-19 wave to the corresponding months in the five years preceding. We considered changes in pneumococcal exposition, delayed or waived referral to the hospital, hampered capacity to diagnose IPD (including prior antibiotics use), and potential adjustments in standards of hospital care as possible explanatory factors. Understanding what sample of adult pneumococcal infections has been captured in the IPD registration is of importance for surveillance purposes as well as for individual case management in subsequent COVID-19 waves.

\section{Altered numbers and mortality in hospital-based survey}

During the first COVID-19 wave in March, April, and May 2020, a total of 13 adults with pneumococcal bacteremia were hospi- 


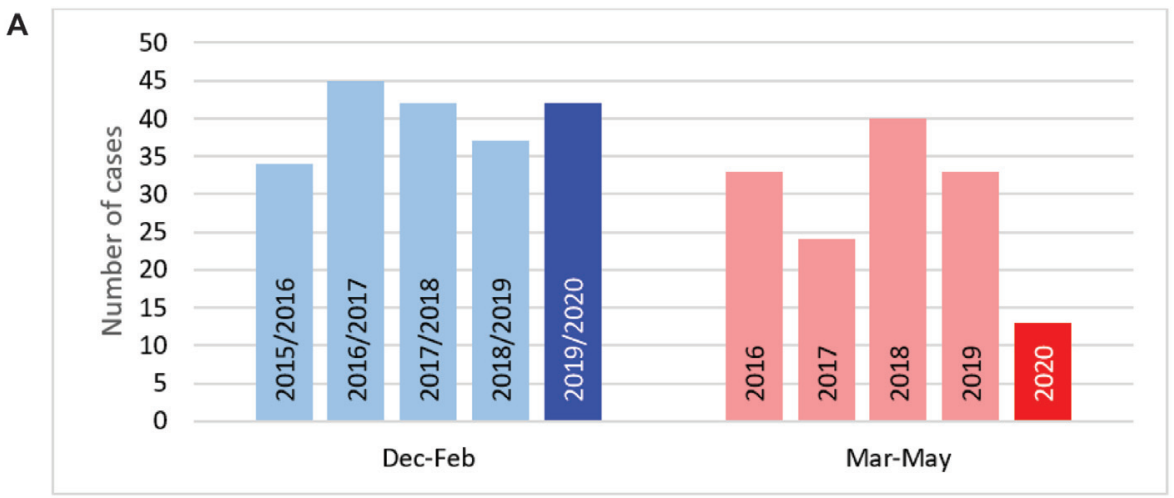

B

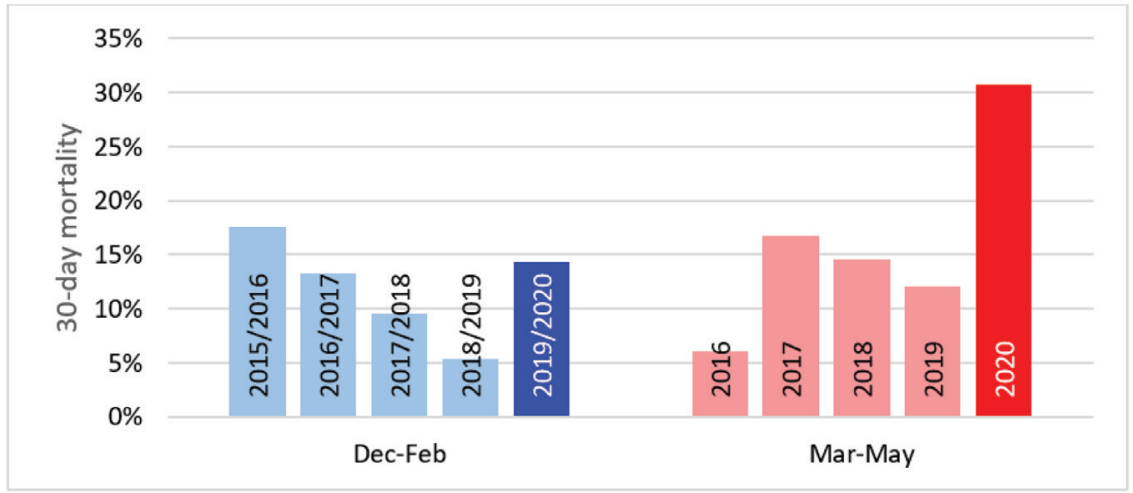

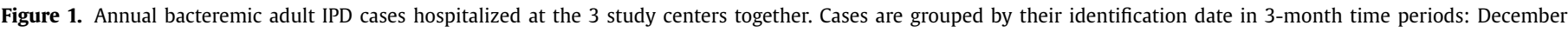
to February or March to May. Displayed are number of cases (panel A) and 30-day mortality (panel B).

talized in the three participating hospitals, compared to $32 \pm 6$ (mean $\pm \mathrm{SD}$ ) cases during the corresponding months in the five years preceding (Figure $1 \mathrm{~A}$ ). Among these 13 cases, 30-day mortality was $30.8 \%$ (four out of 13 ) compared to $9.9 \%$ (16 out of 161 ) in earlier years $(\mathrm{p}=0.046)$ (Figure $1 \mathrm{~B})$.

\section{Ongoing disease burden despite decreased transmission}

Nasopharyngeal pneumococcal carriage is most prevalent among children under the age of five who are the main source of circulating S. pneumoniae (Bogaert et al., 2004, Vissers et al., 2018). By contrast, pneumococcal colonization is only occasionally detected among older adults (Palmu et al., 2012). The timeline in Figure 2 describes measures taken by the national government to control the COVID-19 outbreak in relation to the number of IPD cases identified at the three participating hospitals. During the first COVID-19 wave, daycare and primary schools were closed from mid-March until early May. Social distancing was advocated and included maintaining a 1.5-meter distance, restrictions on crowding, and the elderly were advised to withhold from interaction with their grandchildren. Exposure to pneumococcal carriers likely decreased among the elderly, partly explaining the drop in observed IPD cases.

However, a recent study showed that $32 \%$ of healthy volunteers of 50-84 years old were still colonized by S. pneumoniae four weeks after the experimental challenge (Adler et al., 2020). This suggests that into the COVID-19 pandemic, the elderly who were already colonized by $S$. pneumoniae were still at risk for pneumococcal infection despite social distancing measures. Also, for the IPD cases in May, it is not excluded that they had acquired pneumococci from external pneumococcal carriers long before developing the disease. The presence of high avidity serotype-specific antibodies in the elderly with IPD indicates that weeks of anti- body maturation can occur prior to actual infection (Cremers et al., 2014b). In addition, by molecular methods, S. pneumoniae is still being detected in nasopharyngeal samples after cultures have become negative, and endogenous pneumococcal low-density carriage can again increase over time (Gritzfeld et al., 2014).

In the Netherlands, the prescription of amoxicillin by general practitioners (GPs) is typically intended as the first-line treatment for bacterial pneumonia (Verheij et al., 2011). Data from GPs' pharmacists in the study area demonstrate that the prescription of amoxicillin during the first COVID-19 wave decreased overall by $25 \%$, but the level of prescriptions was sustained among adults 2140 years old and those 75 and over (Figure 3). This could be due to the persistent occurrence of pneumococcal respiratory infections in these age categories. At the same time, age over 75 has been an important reason not to withhold antibiotics as it is a risk factor for bacterial pneumonia, which may be hard to differentiate from COVID-19 according to the Dutch College of General Practitioners (El Bouazzaoui, 2021).

Infections by respiratory viruses like influenza come with a serious risk of bacterial superinfection (McCullers, 2014). However, in our dataset, only one out of 13 adult bacteremia IPD cases was simultaneously diagnosed with COVID-19. In line with that observation, while potential pathogens have been identified in nasopharyngeal specimens of COVID-19 cases (Zhu et al., 2020), actual co-infections are infrequently observed (Karami et al., 2020, Lansbury et al., 2020). SARS-CoV-2 is a less potent inducer of proinflammatory cytokines compared to influenza (Hui et al., 2020). Another explanation for the relatively low number of IPD and COVID-19 co-infections could be the fact that during the first COVID-19 wave, adults mainly contracted SARS-CoV-2 infection from contacts other than children (PosfayBarbe et al., 2020, van der Hoek et al., 2020), thereby, outside of the pediatric population, avoiding concurrent exposure 


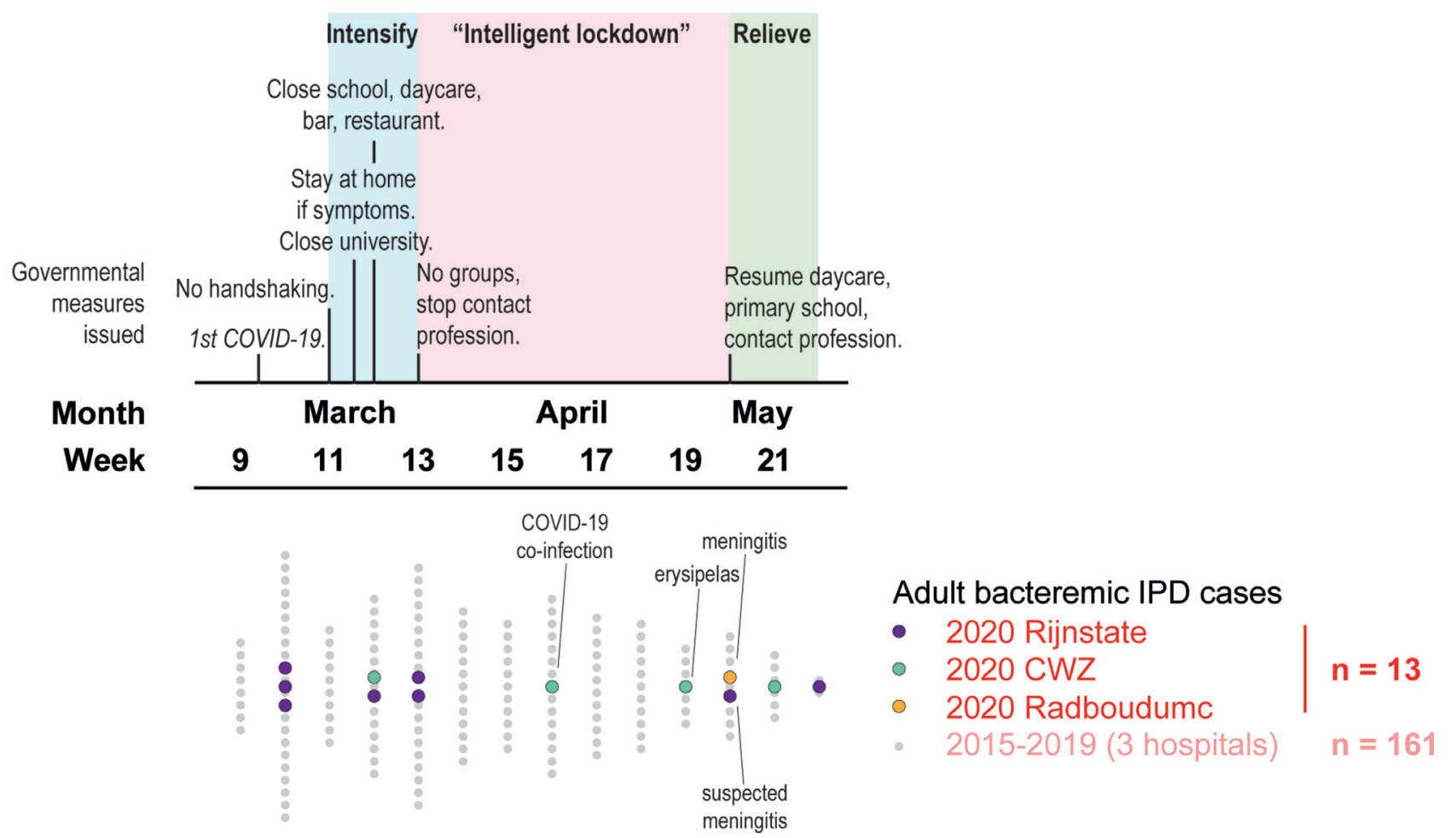

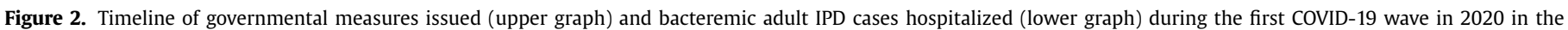
Netherlands.

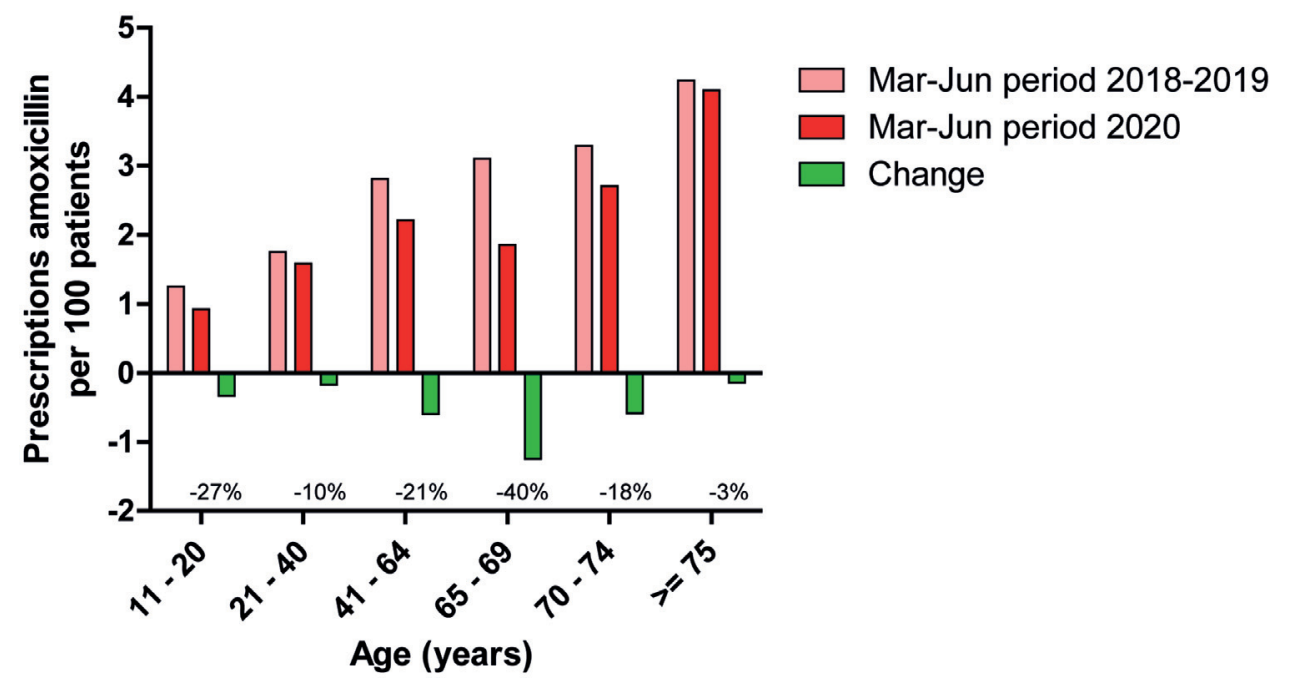

Figure 3. Decreases in the prescription of amoxicillin by GPs in the study area during the first COVID-19 wave, stratified by patient age.

to the main reservoir for bacterial respiratory pathogens like S. pneumoniae.

Taken together, while social distancing measures will indeed have mitigated the transmission of $S$. pneumoniae, a significant amount of infections may still have occurred among adults.

\section{Factors that mediated selective hospital referral}

In the Netherlands, GPs are the primary consultant for medical issues and act as gatekeepers who determine if referral to a hospital or Emergency Department (ED) is indicated. While the number of GP consultations for respiratory infections peaked during our study period, in parallel, patients frequently abstained from seeking medical care or abstained from in-hospital treatment out of fear of SARS-CoV-2 transmission, fear to overburden the health care system, and other personal grounds (Barten et al., 2020, Giessen et al., 2020, Heins et al., 2020). Once referral was desired and indicated, GPs experienced no particular barriers towards ED evaluation during the first wave of COVID-19, yet only part of these evaluations led to the hospitalization and microbiological diagnostic testing (personal communication by co-author Rutten).

Outpatient management of bacteremic pneumococcal pneumonia with oral amoxicillin will often be reasonably adequate in the Netherlands, as pneumococci are generally susceptible. The risk of complications like pleural empyema is around 6\%, however, and, in that case, (prolonged) antibiotic treatment without drainage may be suboptimal. Meningitis is a clinical emergency for which treatment with oral penicillins will most often fail. However, these spe- 


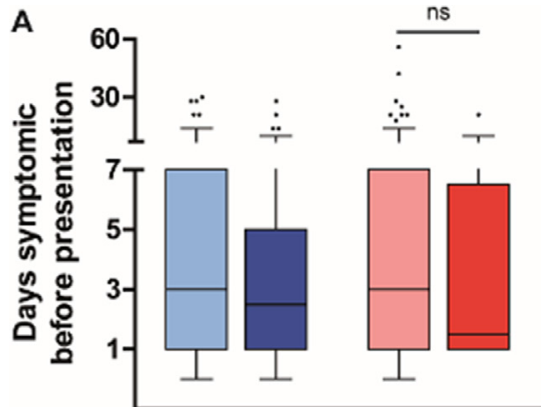

Time period

B

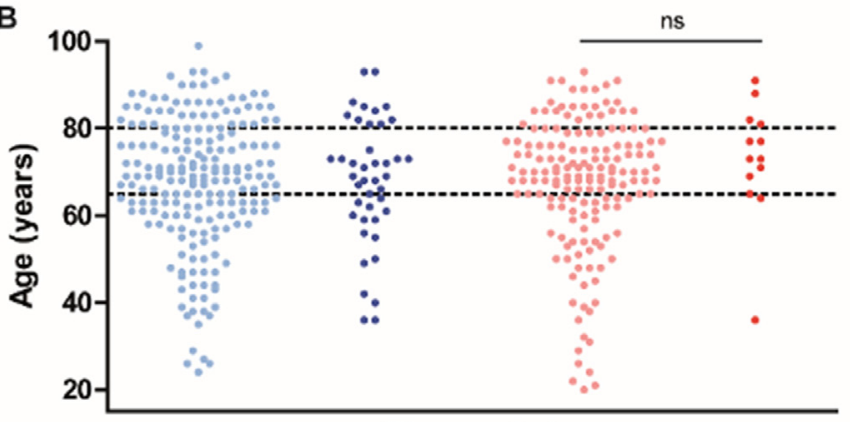

Time period

D

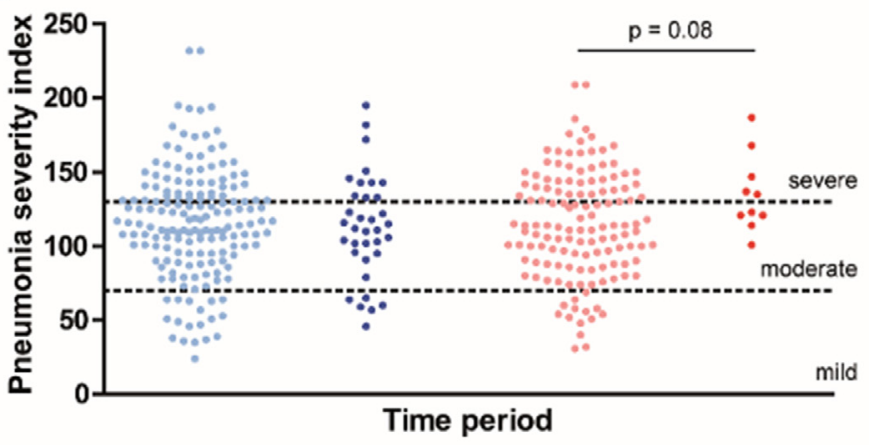

Dec-Feb period in 5 years preceding

Dec-Feb period in 2019-2020

Mar-May period in 5 years preceding

Mar-May period in $2020=$ First wave COVID-19

C
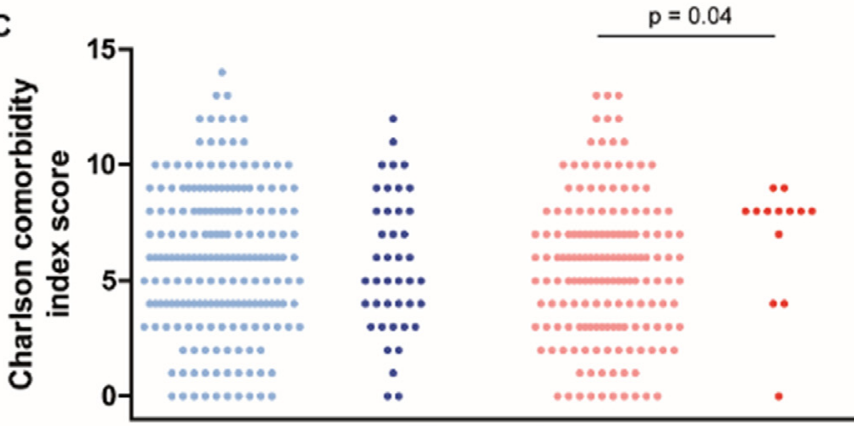

Time period

E

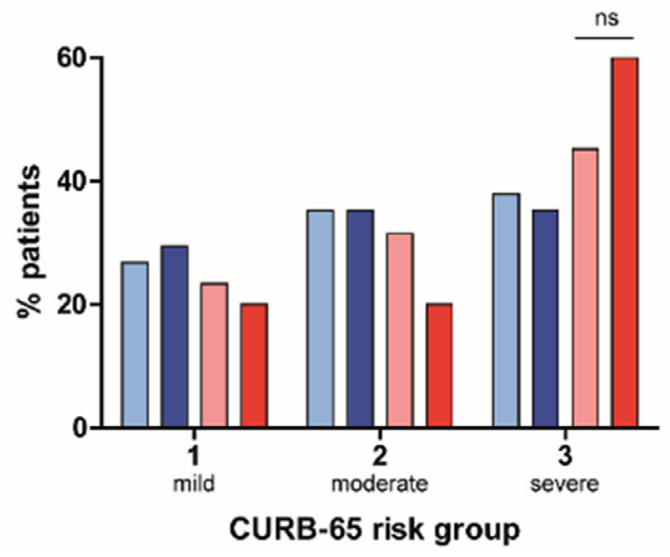

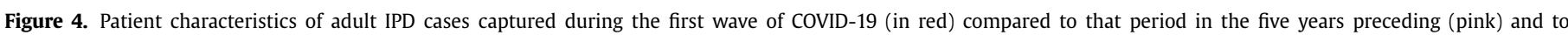

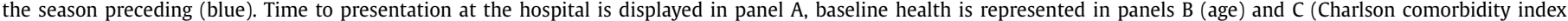
score). Severity of pneumonia cases at the emergency department is shown in panels D (pneumonia severity index) and E (CURB-65). Ns = not significant.

cific complications can be hard to recognize in the outpatient setting. More generally, outcomes for the elderly with moderate to severe disease are likely to be affected by restricted outpatient management. Despite these infection-oriented considerations, assuming that residual IPD has taken place, home treatment seems to have been a deliberate choice as hardly any delayed or complicated admissions with positive blood cultures have been observed.

Clinical diagnoses of the 13 adult pneumococcal bacteremia cases identified during the first COVID-19 wave in the Netherlands showed a regular distribution: ten had pneumonia (three complicated with pleural effusion of which one was proven empyema), and the other manifestations were one proven meningitis, one suspected meningitis without CSF culture performed, and one skin infection. However, several other characteristics suggest that a particular subset of IPD cases was hospitalized. In the months preceding the first COVID-19 wave, obesity was markedly common among adult IPD patients (40\%), while this proportion normalized from March onwards. Figure 4 and Table 1 demonstrate that identified
IPD cases were referred without delay and generally concerned elderly women with comorbidity and severe pneumonia. The proportion of cases with cancer was twice as high compared to earlier years. Eight IPD cases with five different types of solid tumors and hematological malignancies were captured. Significantly fewer IPD patients were eligible for ICU-treatment during the first COVID-19 wave compared to previous years (Table 2). Towards the end of the first COVID-19 wave, early cases in May concerned suspected meningitis cases and erysipelas irresponsive to antibiotic treatment. Only after the relief of specific social distancing measures, bacteremic pneumococcal pneumonia cases reemerged. Therefore, it seems that during the peak of COVID-19, only seriously ill elderly with considerable comorbidity were selected for in-hospital treatment.

Another interesting observation is the earlier decline in IPD cases in hospitals CWZ and Radboudumc, compared to Rijnstate (Figure 1). The first two hospitals are located in Nijmegen 'below the rivers', referring to the dividing line between the North and 
Table 1

Clinical characteristics of adult IPD patients per time period. Data are presented as \% (n) or mean ( \pm standard deviation).

\begin{tabular}{|c|c|c|c|c|c|c|}
\hline & \multicolumn{3}{|l|}{ Dec-Feb period } & \multicolumn{3}{|l|}{ Mar-May period } \\
\hline & $2014-2019 n=202$ & $2019-2020 n=42$ & p-value & $\begin{array}{l}\text { 2015-2019 } n=161 \\
\text { 1st wave COVID-19 }\end{array}$ & $2020 n=13$ & p-value \\
\hline Age (years) & $68 \pm 15$ & $68 \pm 14$ & 0.97 & $67 \pm 15$ & $73 \pm 14$ & 0.20 \\
\hline Gender (female) & $50.0(101)$ & $57.1(24)$ & 0.50 & $46.0(74)$ & $69.2(9)$ & 0.15 \\
\hline Nursing home residence & $1.5(3)$ & $2.4(1)$ & 0.53 & $4.3(7)$ & $0(0)$ & 1 \\
\hline COPD & $22.8(46)$ & $21.4(9)$ & 1 & $20.5(33)$ & $30.8(4)$ & 0.48 \\
\hline Diabetes mellitus type 2 & $21.3(43)$ & $14.3(6)$ & 0.40 & $16.8(27)$ & $15.4(2)$ & 1 \\
\hline Cancer & $39.1(79)$ & $38.1(16)$ & 0.90 & $25.5(41)$ & $61.5(8)$ & 0.01 \\
\hline Body Mass Index & $25.9 \pm 5.4$ & $29.2 \pm 7.5$ & 0.008 & $26.0 \pm 4.8$ & $24.9 \pm 5.7$ & 0.43 \\
\hline Confusion as symptom & $16.8(34)$ & $16.7(7)$ & 1 & $20.5(33)$ & $7.7(1)$ & 0.47 \\
\hline Oxygen saturation (\%) & $93 \pm 5$ & $93 \pm 5$ & 0.27 & $93 \pm 5$ & $93 \pm 6$ & 0.73 \\
\hline Arterial blood $\mathrm{pH}$ & $7.42 \pm 0.10$ & $7.44 \pm 0.07$ & 0.31 & $7.42 \pm 0.08$ & $7.40 \pm 0.13$ & 0.40 \\
\hline C-reactive protein $(\mathrm{mg} / \mathrm{dL})$ & $245 \pm 156$ & $277 \pm 166$ & 0.23 & $249 \pm 161$ & $277 \pm 169$ & 0.55 \\
\hline Antibiotics use within $48 \mathrm{~h}$ preceding admission & $4.5(9)$ & $4.8(2)$ & 1 & $5.6(9)$ & $7.7(1)$ & 0.55 \\
\hline Pneumonia & $84.7(171)$ & $83.3(35)$ & 0.82 & $82.0(132)$ & $76.9(10)$ & 0.71 \\
\hline Empyema & $5.0(10)$ & $7.1(3)$ & 0.47 & $5.6(9)$ & $7.7(1)$ & 0.55 \\
\hline Meningitis & $5.4(11)$ & $11.9(5)$ & 0.16 & $7.5(12)$ & $7.7(1)$ & 1 \\
\hline Unknown focus & $5.4(11)$ & $4.8(2)$ & 1 & $6.3(10)$ & 7.7 (1) & 0.59 \\
\hline
\end{tabular}

Table 2

Aspects of clinical care for adult IPD patients per time period. Data are presented as \% (n).

\begin{tabular}{|c|c|c|c|c|c|c|}
\hline & \multicolumn{3}{|l|}{ Dec-Feb period } & \multicolumn{3}{|l|}{ Mar-May period } \\
\hline & $2014-2019 n=202$ & $2019-2020 n=42$ & $\overline{p \text {-value }}$ & $\begin{array}{l}2015-2019 \mathrm{n}=161 \\
\text { 1st wave COVID-19 }\end{array}$ & $2020 n=13$ & $\overline{p \text {-value }}$ \\
\hline Treatment limitation: no ICU & $10.4(21)$ & $16.7(7)$ & 0.29 & $14.3(23)$ & $38.5(5)$ & 0.04 \\
\hline Admission to ICU & $19.4(39)$ & $21.4(9)$ & 0.83 & $28.0(45)$ & $7.7(1)$ & 0.19 \\
\hline Mechanical ventilation & $10.0(20)$ & $16.7(7)$ & 0.28 & $10.6(17)$ & $7.7(1)$ & 1 \\
\hline 30-day mortality & $10.4(21)$ & $11.9(5)$ & 0.78 & $9.9(16)$ & $30.8(4)$ & 0.046 \\
\hline 30-day mortality within ICU & $28.2(11 / 39)$ & $11.1(1 / 9)$ & 0.42 & $22.2(10 / 45)$ & $100(1 / 1)$ & 1 \\
\hline
\end{tabular}

a Patients who are not eligible for admission to ICU either because of their own choice or because treating professionals considered an ICU admission futile.

South of the Netherlands formed by the Waal and Rhine rivers. The first wave of COVID-19 originated in the South of the Netherlands and made its way upwards consecutively, occupying hospitals' capacity. The observed order in reduction is an indication that restricted healthcare access has contributed to the drop in identified adult IPD cases.

\section{Impediments for diagnostic testing}

During the first COVID-19 wave, the benefit of hospital admission for patients with suspected respiratory infection was stringently assessed by both the GP's and the Emergency Department, and for patients who returned home, generally, no microbiological testing was performed. Although diagnostic capacity was under pressure for hospitalized patients, all three hospitals were able to maintain standard blood culture practices with respect to indication and incubation time. CWZ temporarily went from four to two blood culture bottles per patient as maximum incubator capacity was reached, yet for S. pneumoniae in $90 \%$ of cases, both blood culture sets are positive (Isaacman et al., 1996). At this hospital, the number of adults from whom blood cultures were collected in the Emergency Department from March to May was 45\% higher than the year before (915 in 2019 and 1,328 in 2020). This expansion in diagnostic effort was most prominent for patients 20-60 years old (92\% higher) but also took place for patients over 75 (15\% higher). Relative increases in blood cultures performed at the Emergency Department were more modest at Radboudumc (+ 8\%) and Rijnstate (+ 18\%). S. pneumoniae was cultured from $1.5 \%$ (14/915) versus $0.3 \%(4 / 1,328)$ of blood cultures performed at CWZ during the 2019 and 2020 periods, respectively $(\mathrm{p}=0.018)$. Although blood culture contamination rates were elevated during the first COVID-19 wave in all three hospitals, it is unlikely that this has masked the identification of $S$. pneumoniae as its time to blood culture positivity is generally short. Delays between the collection of blood cultures and the start of incubation do affect the chances of pneumococcal growth (Roback et al., 1994), but this has not been an issue in the participating hospitals with blood culture incubators present within the central hospital building.

Prior use of antibiotics heavily impedes pathogen identification in community-acquired pneumonia (CAP) because it hampers bacterial growth in diagnostic cultures, also when the antibiotic may be unsuited to treat pneumococcal infections (Endeman et al., 2008). S. pneumoniae is especially sensitive to prior antibiotics use, with odds for positive blood cultures dropping to 0.20 , compared to 0.54 for all CAP-causing bacteria (Driscoll et al., 2017). For the Dutch CAP population, antibiotics use within 14 days before hospital presentation is usually around 30\% (Huijts et al., 2013, Postma et al., 2015). However, a random sample of patients presenting with COVID-19 to the study hospitals showed that $40 \%$ had used antibiotics in the past 2 days, amoxicillin allocated to the majority. The high number of COVID-19 patients pre-treated with antibiotics is in contrast to the 13 identified adult IPD cases of whom only one had received antibiotics within 48 hours before presentation, long-term doxycycline prophylaxis to which the pneumococcal isolate was resistant. Furthermore, cerebrospinal fluid cultures are probably less affected by prior oral antibiotics use than blood cultures (Kaplan et al., 1986, Korsgaard et al., 2005). Outside our study population of pneumococcal bacteremia, up to September 2020, three cases of pneumococcal meningitis with negative blood cultures were already established, while this normally occurs about once a year.

Therefore, we presume that more patients with pneumococcal bacteremia did reach the hospital but have not been recognized 
Table 3

Summary of factors that contribute to the reporting of IPD.

\begin{tabular}{ll}
\hline Links in the chain towards capturing IPD & Relevant aspects in the COVID-19 pandemic \\
\hline Transmission of S. pneumoniae & - The extent to which social distancing measures \\
permit interaction among young children and adult & caregivers. \\
& - Attention for hand hygiene. \\
& - Individual nasopharyngeal carriage can persist for \\
& months. \\
& - Individual hesitance to seek healthcare. \\
- Outpatient treatment for IPD often suffices to recover. & - Selection of patients for hospital admission. \\
Docation of care & - Allocation and quality of blood culture practices. \\
& - Pretreatment with antibiotics before admission. \\
& - Reporting to surveillance bodies.
\end{tabular}

as such due to prior use of antibiotics or because of return home without microbiological testing.

\section{Modifications in-hospital care}

A case-control analysis was performed to assess whether 30day mortality among the 13 identified IPD cases actually deviated from expected. Cases were adults with IPD in 2020, who were each matched to four controls from 2015-2019 based on baseline probability of dying from advent IPD (age, comorbidity, focus of pneumococcal infection). The relative risk of being infected during the first COVID-19 wave among deaths compared to survivors was 1.96 (95\% confidence interval: $0.7-5.3 ; p=0.19$ ). Therefore, mortality did not significantly deviate from expected in the IPD sample that was captured. At the same time, hospital care for IPD cases may have been modified during the first wave of COVID-19 due to isolation practices being applied and increased public engagement in treatment restrictions.

Adult IPD cases were not generally notifiable during the study period, yet CWZ and Rijnstate both serve as sentinel laboratories for national IPD surveillance and reported all identified cases within fiv days after blood culture collection, even during the pandemic.

\section{Conclusion}

We studied changes in confirmed cases of adults with pneumococcal bacteremia during the first wave of COVID-19 in three adjacent hospitals in Eastern Netherlands, compared to the five preceding years. We conclude that the drop in identified cases is multifactorial, with likely contributions of repressed referral practices and impaired diagnostic yield, in addition to decreased transmission (Table 3). Expected cases that were not captured compared to the years before are elderly men and the younger population with milder disease. Our data suggest that these patients may have gotten infected but no longer reached the hospital or were not identified due to prior use of antibiotics. The mortality among adult IPD cases that were identified was not higher than expected.

\section{Evaluation and possible solutions}

Illustrative for transmission and being just one of many links in the chain towards capturing IPD is that seven out of eight bacteremic IPD cases with solitary pneumonia in our study had cancer. These patients are more readily admitted to the hospital during the pandemic directly by their attending medical oncologist, without the interference of a GP or prior antibiotics, which increases the chances of IPD being detected. Patients with presumed pneumonia, their GPs, and their assessors at the ED have been more critical towards the desire for in-hospital treatment and often opted for treatment at home in a void of microbiological testing. Such selection bias will flaw surveillance on the level of incidence estimates, but it may also affect the validity of antimicrobial resistance and serotype dynamics.

International IPD surveillance data demonstrated that, after a sharp drop in mobility, IPD incidence rates continued to gradually decrease over months (Brueggemann et al., 2021). This additional attenuation of expected cases could not be attributed to school closures. Also, the transmission of respiratory viruses like influenza that may predispose for IPD ceased instantaneously after the Dutch lockdown, not tapering off like IPD (RIVM, 2020b, Zhou et al., 2012). It may instead represent universal gradual waning of pneumococcal colonization and subsequent disease. Instant drops in IPD reporting are more likely to be related to changes in health care provision and diagnostic yield, and therefore more countryspecific. In the Netherlands, where pneumococci are susceptible to first-line antibiotics, and a strong GP system is in place, the instant decline in captured IPD was probably not just attributable to decreased transmission.

Of particular interest is the comparison between S. pneumoniae and Haemophilus influenzae. These two respiratory pathogens have similar transmission and disease dynamics in the population, yet the incidence of captured invasive $H$. influenzae infections among adults has not decreased during the pandemic in the Netherlands (Middeldorp et al., 2021). The discordance compared to IPD may again be explained by particular Dutch treatment and referral practices. H. influenzae is more steadily cultured (Ling et al., 2021), more often resistant to amoxicillin, and the population at risk for invasive $H$. influenzae infection are adults with humoral immunodeficiencies who are often already in specialist care with hospitalization and microbiological diagnostic testing more readily performed.

To maintain sight of IPD dynamics, there may be ways that are less affected by (future) fluctuations in clinical practices. It could include sampling at alternative health care providers or using alternative diagnostic techniques. Global surveillance of pneumococcal serotypes in IPD is currently based on blood and cerebrospinal fluid cultures. Although the use of pneumococcal urinary antigen tests is under debate because of limited sensitivity (60-75\%) and protracted positivity, their results are not affected by prior antibiotics use and could be generated outside the hospital (Hyams et al., 2020). Moreover, sensitivity issues can be overcome using a CRP threshold above which urinary antigen testing will be representative (Choi et al., 2015, Watanabe et al., 2015). Also, detection of pneumococcal DNA in blood specimens could be an al- 
ternative to establish the presence of pneumococcal bacteremia as the DNA remains detectable for days after initiation of antibiotics and is absent in healthy carriers of S. pneumoniae (Azzari et al., 2011, Cremers et al., 2014a). This diagnostic modality yields additional microbiological diagnoses in CAP patients, especially in cases with prior use of antibiotics in which other tests failed to identify S. pneumoniae (Kleij et al., 2019). For treatment decisions in the GP setting, it is unclear whether the result of either test (urinary antigen and blood DNA) would make a difference. The particular benefit for surveillance purposes is that both can be performed in retrospect on stored specimens. In addition, genotyping pneumococcal DNA from blood can provide information on serotype, virulence factors, and antibiotic resistance markers (Cremers et al., 2019, Elberse et al., 2015). Currently, the Dutch national case definition of notifiable IPD is being extended to adults to monitor the impact of a novel vaccination strategy. Because culture is the gold standard for invasive pneumococcal infections, it is an option to retain hospital-generated culture data as the primary source for monitoring vaccine impact. However, in areas where referral and diagnostic practices are unstable, other diagnostic techniques may provide a valuable alternative to monitor incidence and serotype dynamics of IPD, even in patients who are not hospitalized.

Inherent to the analysis of a reduced number of cases is that the evidence presented in this study is based on inference. Our theory of undetected residual IPD cases requires confirmation by an alternative study design. Furthermore, this study was limited to a restricted region and to adults with positive blood cultures. Therefore, children and rarer cases of IPD with S. pneumoniae cultured solely from a different site of infection were omitted. For adults, however, we think that our in-depth analysis beyond the reported numbers provides a relevant impression and a valuable framework to assess potential causes of decreased reporting of infectious diseases like IPD in times of a pandemic.

\section{Acknowledgments}

We acknowledge all participating hospitals and affiliated researchers for their contribution and support. We thank pharmacist Sjanne van Roijen for sharing the data on antibiotic prescriptions in the GP population.

\section{Conflict of Interest}

The authors declare that they have no known competing financial interests or personal relationships that could have appeared to influence the work reported in this paper.

\section{Funding Source}

This study was initiated by the BACON study group (Bacteremia Collection East Netherlands) as part of a research project on Genomic epistasis of invasive $S$. pneumoniae funded by a Research Grant 2020 from the European Society of Clinical Microbiology and Infectious Diseases (ESCMID) to A.J.H. Cremers.

\section{Ethical Approval}

The study was approved by a central Medical Ethics Committee and the local Ethics Committees of all participating hospitals.

\section{References}

Adler H, German EL, Mitsi E, Nikolaou E, Pojar S, Hales C, et al. Experimental Human Pneumococcal Colonisation in Older Adults is Feasible and Safe, Not Immunogenic. Am J Respir Crit Care Med 2020.

Azzari C, Cortimiglia M, Moriondo M, Canessa C, Lippi F, Ghori F, et al. Pneumococcal DNA is not detectable in the blood of healthy carrier children by real-time PCR targeting the lytA gene. J Med Microbiol 2011;60(Pt 6):710-14.
Barten DG, Latten GHP, van Osch FHM. Reduced Emergency Department Utilization During the Early Phase of the COVID-19 Pandemic: Viral Fear or Lockdown Effect? Disaster Med Public Health Prep 2020:1-4.

Bogaert D, De Groot R, Hermans PW. Streptococcus pneumoniae colonisation: the key to pneumococcal disease. Lancet Infect Dis 2004;4(3):144-54.

Brueggemann $A B$, Jansen van Rensburg MJ, Shaw D, McCarthy ND, Jolley KA, Maiden MCJ, et al. Changes in the incidence of invasive disease due to Streptococcus pneumoniae, Haemophilus influenzae, and Neisseria meningitidis during the COVID-19 pandemic in 26 countries and territories in the Invasive Respiratory Infection Surveillance Initiative: a prospective analysis of surveillance data. Lancet Digit Health 2021;3(6):e360-ee70.

Choi MJ, Song JY, Cheong HJ, Jeon JH, Kang SH, Jung EJ, et al. Clinical usefulness of pneumococcal urinary antigen test, stratified by disease severity and serotypes. J Infect Chemother 2015;21(9):672-9.

Cremers AJ, Hagen F, Hermans PW, Meis JF, Ferwerda G. Diagnostic value of serum pneumococcal DNA load during invasive pneumococcal infections. Eur J Clin Microbiol Infect Dis 2014a;33(7):1119-24.

Cremers AJ, Lut J, Hermans PW, Meis JF, de Jonge MI, Ferwerda G. Avidity of antibodies against infecting pneumococcal serotypes increases with age and severity of disease. Clin Vaccine Immunol 2014b;21(6):904-7.

Cremers AJH, Mobegi FM, van der Gaast-de Jongh C, van Weert M, van Opzeeland FJ, Vehkala M, et al. The Contribution of Genetic Variation of Streptococcus pneumoniae to the Clinical Manifestation of Invasive Pneumococcal Disease. Clin Infect Dis 2019;68(1):61-9.

Driscoll AJ, Deloria Knoll M, Hammitt LL, Baggett HC, Brooks WA, Feikin DR, et al. The Effect of Antibiotic Exposure and Specimen Volume on the Detection of Bacterial Pathogens in Children With Pneumonia. Clin Infect Dis 2017;64(suppl_3):S368-SS77.

El Bouazzaoui L, Bouma M, Cals J, Geersing G, Greving J, Kok-Pigge A, Loogman M, Nijs M, Platteel T, Verheij T. NHG Standard COVID-19. 2021. URL: https://corona. nhg.org/behandeling/. Latest accession date: 18-08-2021.

Elberse K, van Mens S, Cremers AJ, Meijvis SC, Vlaminckx B, de Jonge MI, et al. detection and serotyping of pneumococci in community-acquired pneumonia patients without culture using blood and urine samples. BMC Infect Dis 2015; 15:56.

Endeman H, Schelfhout V, Voorn GP, van Velzen-Blad H, Grutters JC, Biesma DH. Clinical features predicting failure of pathogen identification in patients with community-acquired pneumonia. Scand J Infect Dis 2008;40(9):715-20.

Gezondheidsraad. Vaccinatie van ouderen tegen pneumokokken. 2018. URL: https://www.gezondheidsraad.nl/documenten/adviezen/2018/02/28/ vaccinatie-van-ouderen-tegen-pneumokokken. Latest accession date: 1808-2021.

G.B.D. Lower Respiratory Infections Collaborators. Estimates of the global, regional, and national morbidity, mortality, and aetiologies of lower respiratory infections in 195 countries, 1990-2016: a systematic analysis for the Global Burden of Disease Study 2016. Lancet Infect Dis 2018;18(11):1191-210.

Giessen v, Wit d, Brink vd, Degeling Deuning, Eeuwijk, et al. Impact van de eerste COVID-19 golf op de reguliere zorg en gezondheid. RIVM 2020 URL: https: //www.rivm.nl/bibliotheek/rapporten/2020-0183.pdf, Latest accession date: 18$08-2021$.

Gritzfeld JF, Cremers AJ, Ferwerda G, Ferreira DM, Kadioglu A, Hermans PW, et al. Density and duration of experimental human pneumococcal carriage. Clin Microbiol Infect 2014;20(12):01145-51.

Heins M, Hek K, Hooiveld M, Hendriksen J, Korevaar J. Impact coronapandemie op zorgvraag bij huisartsen. Utrecht: Nivel 2020 URL: https://www.nivel.nl/sites/ default/files/bestanden/1003873.pdf, Latest accession date: 18-08-2021.

Hui KPY, Cheung MC, Perera R, Ng KC, Bui CHT, Ho JCW, et al. Tropism, replication competence, and innate immune responses of the coronavirus SARS-CoV-2 in human respiratory tract and conjunctiva: an analysis in ex-vivo and in-vitro cultures. Lancet Respir Med 2020;8(7):687-95.

Huijts SM, van Werkhoven CH, Boersma WG, Buijs J, Buunk G, Compaijen CJ, et al. Guideline adherence for empirical treatment of pneumonia and patient outcome. Treating pneumonia in the Netherlands. Neth J Med 2013;71(10):502-7.

Hyams C, Williams OM, Williams P. Urinary antigen testing for pneumococcal pneumonia: is there evidence to make its use uncommon in clinical practice? ERJ Open Res 2020;6(1).

Isaacman DJ, Karasic RB, Reynolds EA, Kost SI. Effect of number of blood cultures and volume of blood on detection of bacteremia in children. J Pediatr 1996;128(2):190-5.

Kaplan SL, Smith EO, Wills C, Feigin RD. Association between preadmission oral antibiotic therapy and cerebrospinal fluid findings and sequelae caused by Haemophilus influenzae type b meningitis. Pediatr Infect Dis 1986;5(6): 626-632.

Karami Z, Knoop BT, Dofferhoff ASM, Blaauw MJT, Janssen NA, van Apeldoorn M, et al. Few bacterial co-infections but frequent empiric antibiotic use in the early phase of hospitalized patients with COVID-19: results from a multicentre retrospective cohort study in The Netherlands. Infect Dis (Lond) 2020:1-9.

Kleij A, Zoll J, Coolen J, Vestjens S, Velzen-Blad Hv, Vlaminckx B, et al. Diagnostic blood PCR and metagenomics in community acquired pneumonia. 2019. Oral presentation at NVMM/KNVM Spring meeting 2019.

Klein Klouwenberg PM, Cremer OL, van Vught LA, Ong DS, Frencken JF, Schultz MJ, et al. Likelihood of infection in patients with presumed sepsis at the time of intensive care unit admission: a cohort study. Crit Care 2015;19:319.

Korsgaard J, Moller JK, Kilian M. Antibiotic treatment and the diagnosis of Streptococcus pneumoniae in lower respiratory tract infections in adults. Int J Infect Dis 2005;9(5):274-9. 
Lansbury L, Lim B, Baskaran V, Lim WS. Co-infections in people with COVID-19: a systematic review and meta-analysis. J Infect 2020;81(2):266-75.

Leligdowicz A, Dodek PM, Norena M, Wong H, Kumar A, Kumar A et al. Association between source of infection and hospital mortality in patients who have septic shock. Am J Respir Crit Care Med 2014;189(10):1204-13.

Ling CL, Roberts T, Soeng S, Cusack TP, Dance DAB, Lee SJ, et al. impact of delays to incubation and storage temperature on blood culture results: a multi-centre study. BMC Infect Dis 2021;21(1):173.

McCullers JA. The co-pathogenesis of influenza viruses with bacteria in the lung. Nat Rev Microbiol 2014;12(4):252-62.

Middeldorp M, van Lier A, van der Maas N, Veldhuijzen I, Freudenburg W, van Sorge NM, et al. Short term impact of the COVID-19 pandemic on incidence of vaccine preventable diseases and participation in routine infant vaccinations in the Netherlands in the period March-September 2020. Vaccine 2021;39(7):1039-43.

Palmu AA, Kaijalainen T, Saukkoriipi A, Leinonen M, Kilpi TM. Nasopharyngeal carriage of Streptococcus pneumoniae and pneumococcal urine antigen test in healthy elderly subjects. Scand J Infect Dis 2012;44(6):433-8.

Posfay-Barbe KM, Wagner N, Gauthey M, Moussaoui D, Loevy N, Diana A, et al. COVID-19 in Children and the Dynamics of Infection in Families. Pediatrics 2020;146(2).

Postma DF, van Werkhoven CH, van Elden LJ, Thijsen SF, Hoepelman AI, Kluytmans JA, et al. Antibiotic treatment strategies for community-acquired pneumonia in adults. N Engl J Med 2015;372(14):1312-23.

RIVM. The National Immunisation Programme in the Netherlands Surveillance and developments in 2019-2020. RIVM Report 2020-0077. 2020a. URL: https://www. rivm.nl/bibliotheek/rapporten/2020-0077.pdf. Latest accession date: 18-08-2021.

RIVM. Annual report Surveillance of influenza and other respiratory infections in the Netherlands: winter 2019/2020. RIVM Report 2020-0177. 2020b. URL: https: //www.rivm.nl/bibliotheek/rapporten/2020-0177.pdf. Latest accession date: 1808-2021.

Roback MG, Tsai AK, Hanson KL. Delayed incubation of blood culture bottles: effect on recovery rate of Streptococcus pneumoniae and Haemophilus influenzae type B. Pediatr Emerg Care 1994;10(5):268-72.
Ruiz-Gonzalez A, Falguera M, Nogues A, Rubio-Caballero M. Is Streptococcus pneumoniae the leading cause of pneumonia of unknown etiology? A microbiologic study of lung aspirates in consecutive patients with community-acquired pneumonia. Am J Med 1999;106(4):385-90.

van de Beek D, Brouwer M, Hasbun R, Koedel U, Whitney CG, Wijdicks E. Community-acquired bacterial meningitis. Nat Rev Dis Primers 2016;2:16074.

van der Hoek W, Backer JA, Bodewes R, Friesema I, Meijer A, Pijnacker R, et al. The role of children in the transmission of SARS-CoV-2. Ned Tijdschr Geneeskd 2020;164.

van der Poll T, Opal SM. Pathogenesis, treatment, and prevention of pneumococcal pneumonia. Lancet 2009;374(9700):1543-56.

Verheij TJM, Hopstaken RM, Prins JM, Salomé PL, Bindels PJ, Ponsioen BP, et al NHG Standard Acute cough. 2011. URL: https://richtlijnen.nhg.org/standaarden/ acuut-hoesten\#volledige-tekst. Latest accession date: 18-08-2021.

Vincent JL, Sakr Y, Sprung CL, Ranieri VM, Reinhart K, Gerlach H, et al. Sepsis in European intensive care units: results of the SOAP study. Crit Care Med 2006;34(2):344-53.

Vissers M, Wijmenga-Monsuur AJ, Knol MJ, Badoux P, van Houten MA, van der Ende $A$, et al. Increased carriage of non-vaccine serotypes with low invasive disease potential four years after switching to the 10-valent pneumococcal conjugate vaccine in The Netherlands. PLoS One 2018;13(3).

Watanabe H, Uruma T, Tazaki G, Kikuchi R, Tsuji T, Itoh M. Clinical factors associated with negative urinary antigen tests implemented for the diagnosis of community-acquired pneumococcal pneumonia in adult patients. Med Princ Pract 2015;24(2):189-94.

Zhou H, Haber M, Ray S, Farley MM, Panozzo CA, Klugman KP. Invasive pneumococcal pneumonia and respiratory virus co-infections. Emerg Infect Dis 2012;18(2):294-7.

Zhu X, Ge Y, Wu T, Zhao K, Chen Y, Wu B, et al. Co-infection with respiratory pathogens among COVID-2019 cases. Virus Res 2020;285.

Zorginstituut Nederland en Zinnige Zorg. Screeningsrapport Systematische analyse Infectieziekten Zinnige Zorg. 2019. URL: https: //www.zorginstituutnederland.nl/publicaties/rapport/2019/05/14/ zinnige-zorg-rapport-screeningsfase-infectieziekten. Latest accession date: 18-08-2021. 